\title{
Toothbrushing Monitoring using Wrist Watch
}

\author{
Hua Huang \\ Stony Brook University \\ hua.huang@stonybrook.edu
}

\author{
Shan Lin \\ Stony Brook University \\ shan.x.lin@stonybrook.edu
}

\begin{abstract}
Daily toothbrushing is essential for maintaining oral health. However, there is very limited technology to monitor the effectiveness of toothbrushing at home. In this paper, a system is built to monitor the brushing quality on all 16 tooth surfaces using a manual toothbrush and an off-theshelf wrist watch. The toothbrush is modified by attaching small magnets to the handle, so that its orientation and motion can be captured by the magnetic sensor in the wrist watch. The toothbrushing gestures are recognized based on inertial sensing data from the wrist watch. As the acoustic signal collected from the watch is correlated with the motion of toothbrushing stroke, acoustic sensing algorithm is designed to assist in recognition. User-specific toothbrushing order is also utilized to improve the surface recognition. In extensive experiments with 12 users over 3 weeks, our system successfully recognized toothbrushing gestures with an average precision of $85.6 \%$.
\end{abstract}

\section{CCS Concepts}

-Applied computing $\rightarrow$ Life and medical sciences; -Human-centered computing $\rightarrow$ Ubiquitous and mobile computing systems and tools;

\section{Keywords}

Toothbrushing Monitoring, Activity Recognition, Wearables

\section{INTRODUCTION}

Dental caries and gum diseases are among the most prevalent chronic diseases in both children and adults [13]. Most of these dental diseases are the results of bacteria deposited on the surface of the teeth. Without proper brushing, such bacteria accumulate on the tooth surfaces in a complex called plaque, destroying the outermost layer of the tooth (enamel) and initiating gingival inflammation, resulting in dental decay and gum diseases [6]. Proper toothbrushing is crucial to

Permission to make digital or hard copies of all or part of this work for personal or classroom use is granted without fee provided that copies are not made or distributed for profit or commercial advantage and that copies bear this notice and the full citation on the first page. Copyrights for components of this work owned by others than ACM must be honored. Abstracting with credit is permitted. To copy otherwise, or republish, to post on servers or to redistribute to lists, requires prior specific permission and/or a fee. Request permissions from permissions@ acm.org.

SenSys '16, November 14-16, 2016, Stanford, CA, USA

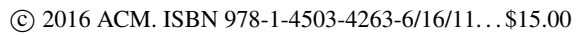

DOI: http://dx.doi.org/10.1145/2994551.2994563 plaque reduction and dental pathology prevention. American Dental Association recommends brushing teeth for two minutes and twice a day using the Bass technique [29, 16]. Unfortunately, there is limited technology for assessment and reassurance of home toothbrushing compliance. There are electric toothbrushes which are designed to make toothbrushing easier and simpler, but they still rely on users to move the toothbrush on the correct tooth surfaces. Also, they do not detect whether the user has brushed with the proper technique, complete coverage and sufficient duration for all tooth surfaces. Therefore, it is desirable to develop technologies for toothbrushing monitoring.

Wearable devices have been employed in recognition of various activities, such as hand-washing [14], smoking [27], and exercising [11]. These research studies show valuable results that demonstrate the potential of activity recognition using wearables in health applications. Different from these applications, toothbrushing requires the usage of a hand tool: the toothbrush. Both the motions of the toothbrush and the user gestures to operate the toothbrush need to be recognized.

The toothbrushing activity consists of a series of gestures for maneuvering a toothbrush to scrub teeth. There are many dynamic interactions between the toothbrush and the user's hand during toothbrushing, such as grabbing, pointing, rotating, and flipping of the toothbrush. As the motions of toothbrush and user gestures are tightly coupled with each other, it is very challenging to model and monitor these activities. In this paper, we present a system for toothbrushing monitoring. This system consists of a manual toothbrush and a wrist watch. The manual toothbrush is modified by attaching small magnets ( 7 cents) on the handle. Then we develop a magnetic sensing algorithm on the wrist watch to capture the orientation of the toothbrush and its relative movement to the hand.

To recognize toothbrushing gestures, we construct toothbrushing gesture models using inertial sensing data. Our model is based on underlying basic motions (wrist flexion/ extension, forearm rotation, elbow flexion/extension, and shoulder flexion/extension), which allows us to characterize each gesture quantitatively by its motion direction, speed, amplitude, and frequency. Besides using inertial sensing data, acoustic data collected from the microphone in the wrist watch is used to recognize toothbrushing gestures as well as toothbrushing stroke frequencies. We also develop a hidden Markov model to learn the order of toothbrushing surfaces, which varies from person to person. By integrating these gesture recognition techniques with the toothbrush 


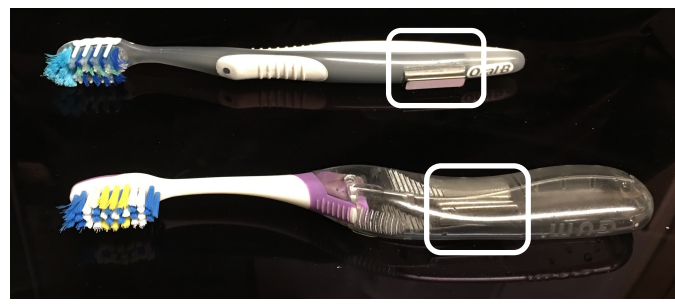

Figure 1: Modified Toothbrush Prototypes

orientation detection, our system is able to recognize the tooth surface being brushed at each moment, and estimate the toothbrushing effectiveness by counting the number of strokes.

We deploy our system with a cloud-based framework, each wrist watch in our system is connected with the backend system on the cloud. 12 users used our system to conduct daily toothbrushing for up to 3 weeks. Experiments have been designed to evaluate the recognition accuracy of different gestures for brushing 16 tooth surfaces, and frequency of strokes. Experimental results demonstrate that our system achieves high recognition accuracy in every toothbrushing session. We also present a case study with 12 users to demonstrate the plague reduction under proper toothbrushing using our system.

We build a real-time toothbrushing monitoring system using wrist watch, which recognizes the brushing quality on all 16 tooth surfaces.

- To capture dynamic interactions between the toothbrush and the userâẮ́s hand, we attach small magnets on off-the-shelf toothbrushes and design novel magnetic sensing algorithms to detect toothbrush orientation and motion.

- We identify coupled motions of a userâĂŹs wrist, hand, and arm during toothbrushing with the Bass technique, and design models based on inertial sensing data to characterize toothbrushing gestures.

- We employ a set of features including order of brushing to improve the recognition performance. We also use acoustic profile to estimate the brushing quality.

- We build a toothbrushing monitoring system prototype using wrist watch. In a 3 week experiments with 12 users, our system successfully recognized the brushing gestures on 16 tooth surfaces with an average precision of $85.6 \%$.

\section{SYSTEM OVERVIEW}

\subsection{System Design and Consideration}

Our system includes the following four components:

- Modified toothbrush. We modified off-the-shelf manual toothbrushes by attaching an array of magnets on the handle of the brush. A picture of such toothbrushes is shown on the Figure 1.

- Wrist watch. We use off-the-shelf wrist watches. Four types of sensors in the wrist watch are used: accelerometer, gyroscope, magnetic sensor, and acoustic sensor. Most signal processing and machine learning algorithms are executed on the smartwatch to ensure realtime processing and to reduce wireless communication traffic. The main idea of the recognition algorithm is demonstrated in Figure 2.

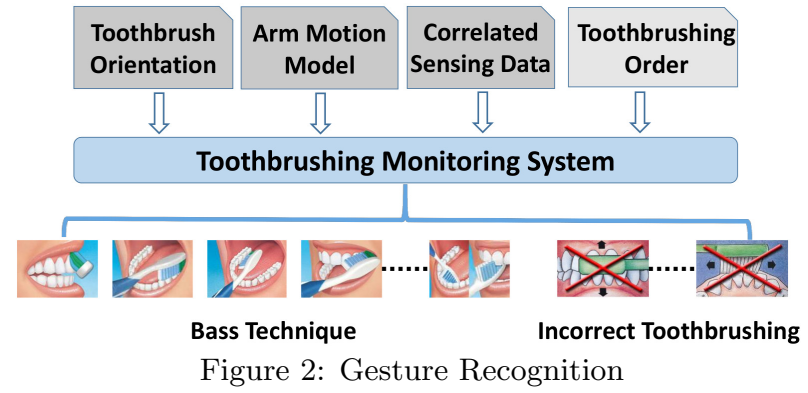

- Smartphone or tablet. A smartphone or tablet is used as the user interface. It also relays data from the wrist watch to the cloud. The real-time brushing surface recognition result is displayed on the phone or tablet.

- Cloud. The cloud stores a database of labeled training data and features, and uses these data to compute a personalized machine learning profile for each user. The cloud also stores the public profile and toothbrushing progress report of the user, so that the user's dentist can monitor the dental hygiene results remotely.

Our system has two phases: the training phase and the running phase. In the training phase, a user is instructed to conduct toothbrushing using the Bass technique with our system for one or multiple times. The system records training data as the baseline. In the running phase, the user conducts daily toothbrushing with our system. Our system recognizes the tooth surface being brushed and detects incorrect toothbrushing techniques in real-time. When the toothbrushing is finished, the system provides a brushing quality report on the number of brushing strokes for each surface of the teeth.

\subsection{The Bass Toothbrushing Technique}

There are 16 major surfaces of the teeth that we brush, as shown in Figure 3. The labial and buccal surfaces are sides of the teeth that are adjacent to the inside of the lips or the cheek. In this paper, we refer them as outer surfaces. The occlusal surfaces of the teeth are the surfaces that come in contact with those in the opposite jaws during biting or chewing. We refer them as chewing surfaces. The palatal and lingual surfaces of the teeth are those next to one's tongue. We refer them as the inner surfaces of the teeth.

For manual brushing, systematic reviews have shown that the Bass technique has consistently demonstrated good results. It allows the toothbrush bristles to reach the interproximal areas and achieves more effective control in plaque levels $[29,16]$. Several basic gestures of the Bass technique are shown at the left lower corner of Figure 2. The general descriptions of the Bass technique are: a) to brush front outer, back outer and back inner surfaces, place the toothbrush at a 45-degree angle to the gums. Brush these areas with vertical movement of the toothbrush bristles. Each brushing stroke begins from the gum line to the tip of the teeth. b) To brush chewing surfaces, move the bristles of the toothbrush along the chewing surface of the back teeth in a back and forth motion. c) To brush front inner surfaces, tilt the brush vertically and make up-and-down strokes.

On the other hand, incorrect brushing, such as the horizontal brushing on the inner or outer surfaces, as shown at the right lower corner of Figure 2, can cause two to three times more wear to the enamel than the Bass technique [7, $17,26]$. 


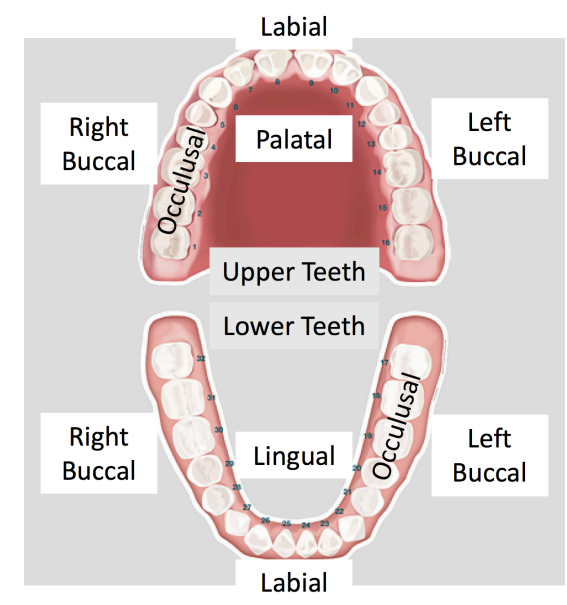

Figure 3: 16 Tooth Surfaces

Sufficient toothbrushing time is also crucial to good toothbrushing results. Many previous oral hygiene studies recommend two minutes of toothbrushing time [15]. However, sufficient overall duration does not guarantee sufficient coverage for all surfaces of the teeth. In practice, people often distribute brushing time unevenly to different surfaces, which may cause over-brushing or under-brushing on some surfaces of the teeth. By monitoring the toothbrushing gestures, our system is able to recognize the toothbrushing duration and the number of brushing strokes for each surface, as well as the overall toothbrushing time.

\subsection{The Coordinate Systems}

We analyze the human arm motions for each toothbrushing gesture quantitatively using a simplified human arm model, as shown in Figure 4. The arm is divided into the upper arm, the forearm, and the hand. The elbow joint connects the upper arm and the forearm; the wrist joint connects the forearm and the hand. The toothbrush is held by the user in his or her hand. Here we use gesture to refer to the movements of the user's arm.

To describe the toothbrush gesture accurately, it is necessary to define the relevant coordinate systems, as shown in Figure 4. The user's coordinate system is denoted by $X_{u}$, $Y_{u}, Z_{u}$. The $X_{u}-Y_{u}$ plane is horizontal to the floor, and the $Y_{u}$ axis points to the facing direction of the user. The sensor data obtained from the watch uses watch coordinates: $X_{w}$, $Y_{w}, Z_{w}$. When the watch is worn on the right wrist, the $X_{w}$ axis is parallel to the forearm. The $Y_{w}$ axis is perpendicular to the forearm and is parallel to the palm, and the $Z_{w}$ axis is perpendicular to the forearm and points toward the back of the forearm. The motion of the toothbrush is described in the toothbrush coordinate system: $X_{t}, Y_{t}, Z_{t} . X_{t}$ axis points to the orientation of the toothbrush bristles, and $Z_{t}$ axis points to the top of the toothbrush. These coordinate systems are used in the following sections.

\section{TOOTHBRUSH BRISTLE ORIENTATION RECOGNITION}

The detection of the motions of the toothbrush in a user's hand is essential for toothbrushing gesture recognition. Specifically, toothbrush motions are critical for gesture recognition in the following scenarios:

- Scenario 1: The toothbrush bristle orientation, i.e.,

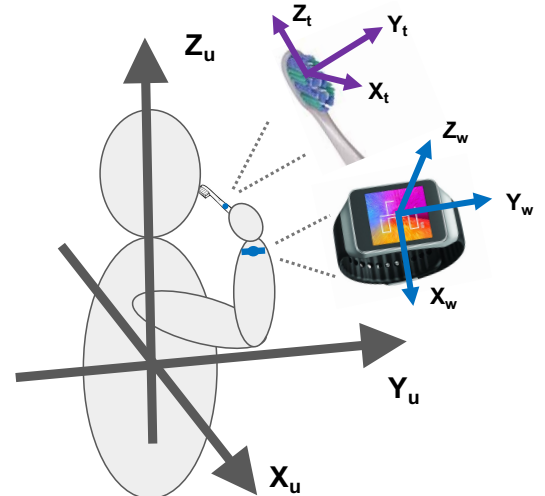

Figure 4: The Arm Model and Coordinate Systems

the orientation of $X_{t}$, as shown in Figure 4. Certain gestures are very similar to each other, such as the ones when brushing the chewing surfaces of the upper and lower teeth. The only difference is the orientations of the toothbrush bristles.

- Scenario 2: Toothbrush rotations around the $Z_{t}$ axis. Certain users use his or her fingers to rotate the toothbrush to scrub teeth.

In our study, we found that these two types of toothbrush motions made almost no effect on wrist-worn accelerometers and gyroscopes. Therefore, it is necessary to apply alternative approaches to detect these motions.

Our solution is to customize off-the-shelf toothbrushes so that they become sensible to the smartwatches. We propose to install small magnets on the toothbrushes. When the magnets move along with the toothbrush, the surrounding magnetic field is disturbed, which can be reliably captured by the magnetic sensor in the watch. This enables our system to recognize the toothbrush motions.

Figure 1 shows a couple prototype toothbrushes. The magnets are marked by white boxes in the figure. We can see that small magnets are attached to the handle of the gray toothbrush, whereas some magnets are put inside the handle of the other purple toothbrush. We tested and found that when the magnet's north pole direction is perpendicular with the $Z_{t}$ direction of the toothbrush, the sensing results were more effective in distinguishing toothbrush bristle orientations. Such small magnets cost only 7 cents each. Our volunteers did not experience any inconvenience with the modified toothbrushes. In the future, we can develop more user-friendly designs, such as a magnetic snap or case that can be easily installed on the handle of a toothbrush.

We denote the magnetic moment of the magnet on the toothbrush as $\vec{m}$, the location of the magnet relative to the watch as $\vec{r}$, and the magnetic permeability of air as $\mu_{0}$. Then the magnetic flux density $\vec{B}=\left(B_{x}, B_{y}, B_{z}\right)$ at the wrist watch is represented by the following equation:

$$
\vec{B}=\frac{\mu_{0}}{4 \pi} \frac{3 \vec{r}(\vec{r} \cdot \vec{m})-\vec{m}|\vec{r}|^{2}}{|\vec{r}|^{5}} .
$$

From the above equation, we can see that when the user changes his or her hold of the toothbrush, which affects $\vec{r}$, or rotates the toothbrush with fingers, which affects $\vec{m}$, the magnetic field $\vec{B}$ will change as well. This enables us to infer toothbrush motions from the magnetic field.

\subsection{Toothbrush Bristle Orientation}

In an experiment, a user held a toothbrush to let the toothbrush bristle face the following 4 surfaces: the Left 


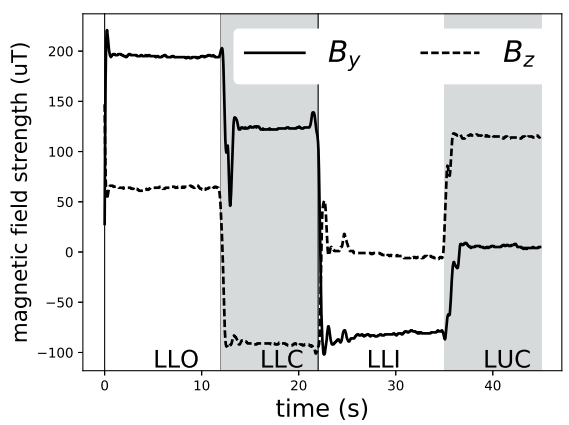

(a) Toothbrush Bristle Orientation

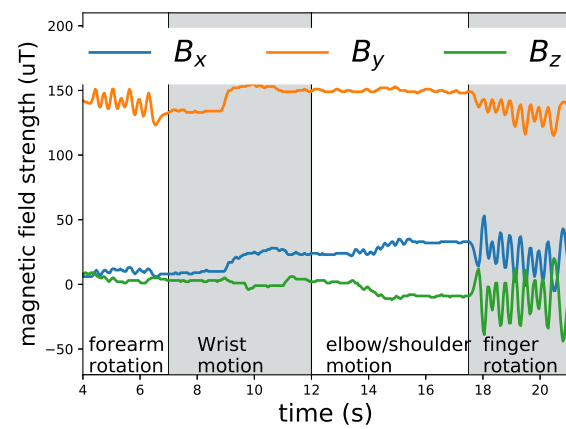

(b) Magnetic Sensing Data under Tooth-(c) Impact of Geo-magnetic Field on Brush brushing Gestures

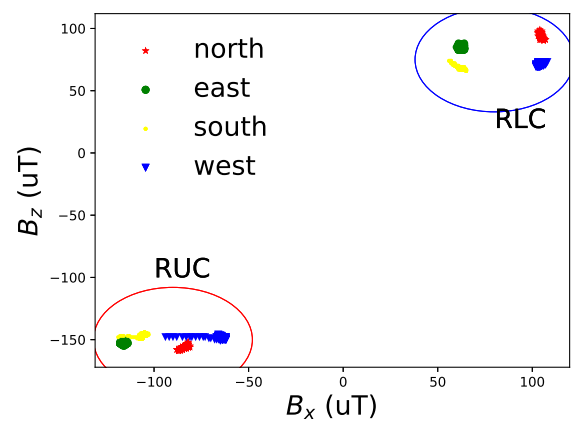

Motion Detection

Figure 5: Magnetic Sensing Results

Lower Outer (LLO) surface, the Left Lower Chewing (LLC) surface, the Left Lower Inner (LLI) surface, and the Left Upper Chewing (LUC) surface, while magnetic sensing data was collected from the wrist watch.

The magnetic sensing result is shown in Figure 5a. From this figure, we can see that the magnetic field strength $B_{y}$ and $B_{z}$ are dramatically different when the toothbrush bristles have different orientations. This allows us to recognize the approximate orientation of the toothbrush bristles during the toothbrushing of each surface. When the toothbrush bristles orientation stays fixed in one direction, the magnetic strength readings are very stable. When the toothbrush bristles orientation changes by 90 degree, the magnetic strength readings change significantly to new levels. This observation suggests us to design a threshold based detection algorithm to capture the toothbrushing surface transitions.

\subsection{Toothbrush Rotation}

We conducted experiments to study the relations between the magnetic sensor data and different toothbrushing motions of the user. In this experiment, a user first performed three basic gestures while holding the toothbrush: the forearm rotations, the wrist flexions/extensions and the elbow/ shoulder motions. After that he rotated the toothbrush with his fingers.

The corresponding magnetic field readings are shown in Figure 5b. From this figure, we can see that when the user is rotating the toothbrush with fingers (from $17 \mathrm{~s}$ to $21 \mathrm{~s}$ ), the magnetic field readings along all the three axes oscillate, with a frequency directly related to the rotation speed. When the user is conducting wrist or elbow/shoulder motions (from $7 \mathrm{~s}$ to $17 \mathrm{~s}$ ), the motions' influences on the magnetic field reading are small, and we don't see any oscillation. This is because neither the relative location of the toothbrush $\vec{r}$, nor the relative direction of the magnetic moment $\vec{m}$ to the watch changes significantly.

\subsection{Robustness to Geomagnetic Field}

The geomagnetic field is the magnetic field that extends from the Earth's interior out into space. It can interfere with our magnetic sensing system and significantly degrade the sensing performance. In our observation, we find that the geomagnetic field has a varying magnitude depending on locations, and its value can go up to more than $100 \mu T$. To obtain robust sensing results under such interference, we use multiple magnets with strong magnetization together. To test the influence of geomagnetic field, we conducted an experiment: a user brushed his Right Lower Chewing (RLC) surface and Right Upper Chewing (RUC) surface with a toothbrush with four small magnets attached, while facing four cardinal directions: east, west, north, and south. We plot the magnetic sensing data of $B_{x}$ and $B_{z}$ axes in the Figure 5c. Clearly, we can see two clusters representing two toothbrush bristles orientations, and these two clusters are separated with a long distance. This indicates that with strong magnets on the toothbrush, we can obtain robust sensing results on the toothbrush bristle orientation, regardless of which cardinal direction the user is facing. We can also see that within a cluster, plotted points with different cardinal directions do not overlap with each other, which shows there is still noticeable impact from the geomagnetic field.

\subsection{Feature Design}

Based on these observations, we design signal processing algorithms to extract features. Given the magnetic field reading in a time window, we firstly calculate average and variance values of $B_{x}, B_{x}$, and $B_{z}$. We also calculate the zero crossing rate, which is defined as the difference between two consecutive times when the magnetic field strength value crosses the average value. In our experiments, when the user is brushing using finger rotations, the zero crossing rate ranges from 0.2 seconds to 1 seconds, with variation less than 3 .

\section{TOOTHBRUSHING GESTURE RECOGNITION}

In this section, we study the motions of the user's arm during the toothbrushing process, and develop algorithms to recognize the gestures for brushing different tooth surfaces. There are two major challenges in achieving this goal:

- Recognition of similar toothbrushing gestures. Effective features need to be designed to capture the subtle differences in gestures of brushing different tooth surfaces.

- Robust recognition with gesture variations. When brushing the same surface, the users can have different arm postures, such as either holding the arm horizontally or vertically. This is a commonly seen variation and can significantly affect the sensing results.

We address the first challenge in Section 4.1 and 4.2 by developing motion models for the arm, and design features based on these models. We address the second challenge in Section 4.3 by studying the user gesture variations and designing a transformation algorithm to offset the effect. 

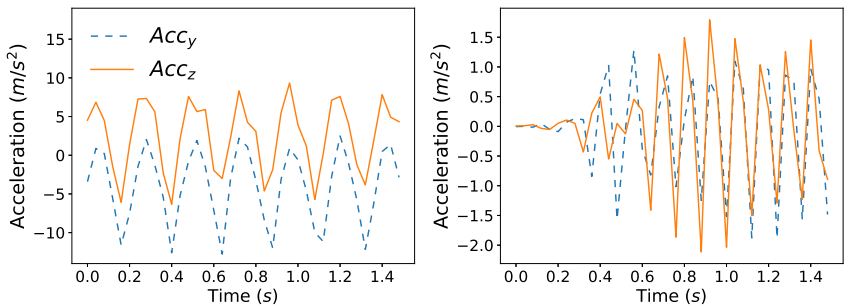

(a) Acceleration Data of El-(b) Band-pass Filtered bow/Shoulder Motion Acceleration data of Elbow/Shoulder Motion
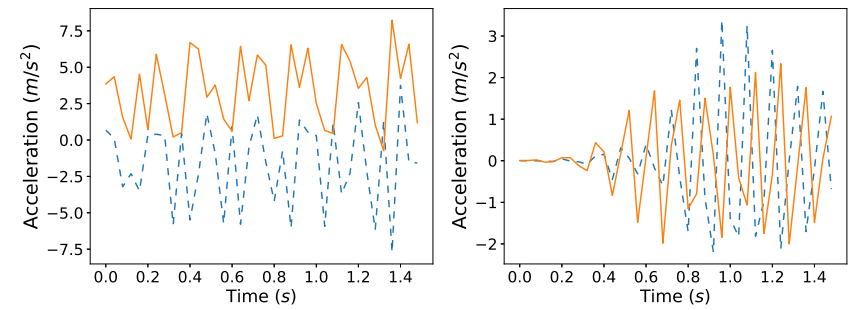

(c) Acceleration data of Wrist(d) Band-pass Filtered AccelMotion eration data of Wrist Motion

Figure 6: Acceleration Data of Elbow and Wrist Motions

\subsection{Toothbrushing Gesture Model}

Although toothbrushing involves complex motions of the human arm, there are four basic types of motions: wrist flexion/extension, forearm rotation (pronation/supination), elbow flexion/extension, and shoulder movement. Each brushing gesture is a combination of these basic motions. Our strategy is to establish models for these motions, and design recognition algorithms based on these models.

We note that both elbow and shoulder movements affect the entire forearm, the wrist watch cannot differentiate these two types of basic motions. So we combine them as a single motion type, the shoulder/elbow flexion/extension.

\subsubsection{Forearm Rotation}

The forearm rotation motion is closely correlated with the gyroscope reading $g_{x}$ along $X_{w}$ axis. This is because the watch is usually tightly attached to the user's wrist, and rotates at the same rate as the forearm. Therefore, we calculate the moving variance of $g_{x}$, denoted by $\sigma_{x}^{g}$, as a feature to indicate the existence of this motion. Our experimental results show that this feature is effective. For example, for many users, the gesture of brushing of left lower outer surface of teeth involves much forearm rotation, and the value of $\sigma_{x}^{g}$ remains large during this brushing gesture.

\subsubsection{Elbow/Shoulder Flexion/Extension}

When a user brushes the two front inner surfaces and the four chewing surfaces, the elbow/shoulder flexion/extension is used primarily. In Figure $6 \mathrm{a}$, we plot the accelerometer data collected when the user is brushing the right lower chewing surface. In this figure, the blue dashed line and the red thick line represent the acceleration of the watch along the $Y_{w}$ axis $\left(A c c_{y}\right)$ and the $Z_{w}$ axis $\left(A c c_{z}\right)$, respectively. We can see that the values of $A c c_{y}$ and $A c c_{z}$ fluctuate periodically according to each brushing stroke. The same pattern can be found when the user is brushing all the other surfaces involving the same basic motion type. This pattern is

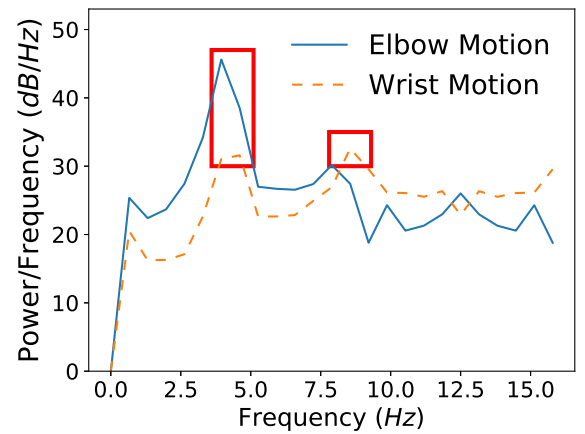

Figure 7: Power Spectrum of Elbow and Wrist Motion Acceleration Data

generated by the regular back-forth horizontal motions. To capture this type of motion, we analyze the power spectrum of the signal. In Figure 7, we plot the log-scale power spectra for the acceleration data of the shoulder/elbow motion (the blue line) and the wrist motion (the red dashed line). From this figure, we can see that for the elbow motions, there is a large peak located at around $4.5 \mathrm{~Hz}$, which corresponds the brushing stroke frequency. Based on this observation, we extract the peak value of the power spectrum $\max \left(P_{a c c}\right)$ and the percentage of energy concentrated around the peak frequency $\left(\operatorname{perc}_{Y}\right)$ as features to recognize this type of motion.

\subsubsection{Wrist Flexion/Extension}

We discover that a characterizing pattern of such motions is the negative correlation between the acceleration values of $A c c_{y}$ and $A c c_{z}$. This is illustrated in Figure 6c, where $A c c_{y}$ (the blue dashed line) and $A c c_{z}$ (the red line) are negatively correlated. This is significantly different from the elbow brushing motions when $A c c_{y}$ and $A c c_{z}$ are positively correlated (shown in Figure 6a). This observation motivates us to measure the correlation of $A c c_{y}$ and $A c c_{z}$ as a feature to recognize the wrist motions.

We also find that in some cases, the user's toothbrushing gestures include both the wrist and the elbow motions. In these cases, both types of motion influence the correlation between $A c c_{y}$ and $A c c_{z}$, and may entirely cover up the wrist motion pattern. This affects the detection accuracy for the system, so it is crucial to separate the influence of these two types of motion.

We notice that for the wrist motions, two peaks are generated in the power spectrum, as shown in the red dashed line in Figure 7. On the other hand, only one peak is generated for the power spectrum of elbow motions (the blue line). This indicates that these two types of motions may create influence on wrist acceleration data at different frequencies. Therefore, we conduct a band-pass filter centering at about two times of regular brushing frequency on the acceleration values, and the results are shown in Figure $6 \mathrm{~d}$. We can see that this filter successfully suppresses the influence of the elbow motion on the correlation between $A c c_{y}$ and $A c c_{z}$, and the feature for wrist motions is revealed. On the other hand, in Figure $6 \mathrm{~b}$, the correlation between $A c c_{y}$ and $A c c_{z}$ is positive due to the absence of wrist motions. Therefore, we will apply a band-pass filter to the acceleration value before calculating the correlations.

The feature to recognize wrist motions is defined as follows. After the acceleration values of $A c c_{y}$ and $A c c_{z}$ are 


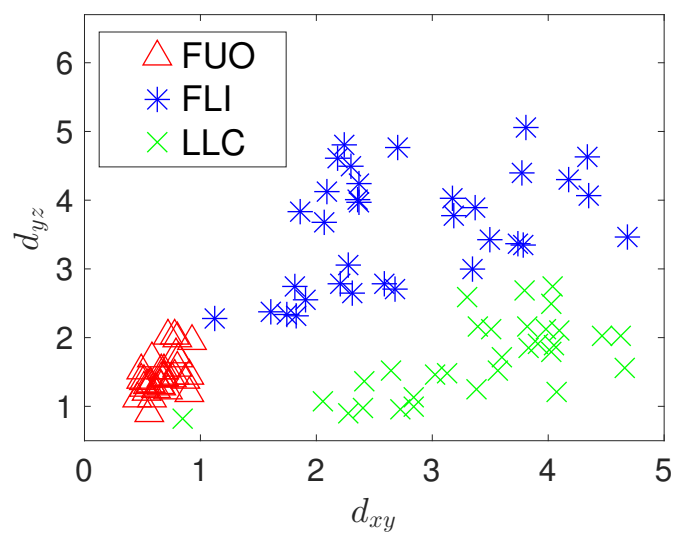

Figure 8: PCA Direction Feature

collected, we apply a band-pass filter with center frequency about two times of brushing frequency, and get the filtered value $A c c_{y}^{\prime}$ and $A c c_{z}^{\prime}$. Then the correlation $C_{y, z}$ between $A c c_{y}^{\prime}$ and $A c c_{z}$ is calculated. If $C_{y, z}$ is smaller than a negative threshold, then wrist motion is detected.

\subsection{Other Features}

\subsubsection{PCA-based motion direction}

When brushing different surfaces, the toothbrush is moving along different directions. For example, when a user brushes his or her chewing surfaces, the toothbrush typically moves along a direction perpendicular to the direction of gravity. On the other hand, when the outer surfaces are being brushed, the toothbrush generally moves along a direction parallel to the direction of gravity. Therefore, the moving direction of the toothbrush also provides important information for the toothbrushing gesture recognition.

We design the Principle Component Analysis (PCA) based motion direction feature to capture this information. PCA [18][5] is a classical algorithm that finds out a set of linearly uncorrelated variables called principal components. The principle components are found in such a way that the first principal component has the largest possible variance, and each succeeding component, in turn, has the highest variance possible under the constraint that it is orthogonal to all the previous ones. In our scenario, the first principal component represents the dominant motion direction of the watch.

Specifically, we calculate the PCA direction feature as follows. For each time window, this feature is extracted from the accelerometer sensor data $\left[A c c_{x}, A c c_{y}, A c c_{z}\right]$. For each pair of dimensions $i$ and $j, i, j \in\{x, y, z\}, i \neq j$, we feed the sensor data $X=\left[A c c_{i}, A c c_{j}\right]$ to the PCA algorithm and compute the eigenvectors $W$ of the covariance matrix $X^{T} X$. The $2 \times 2$ matrix $W$ contains the information about the principle component. We record the slope $d_{i j}=W[2,1] / W[1,1]$ to represent the motion direction in the $i j$ plane.

We plot the PCA direction features for brushing Front Upper Outer (FUO), Front Lower Inner (FLI) and Left Lower Chewing (LLC) surfaces in Figure 8. We can clearly see the plotted points naturally form three clusters, which allows us to differentiate these different gestures. This shows that the PCA direction feature can distinguish not only horizontal (LLC) motions from vertical motions (FUO), but also horizontal motions with different directions (LLC and FLI).

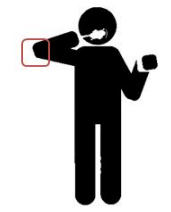

(a)

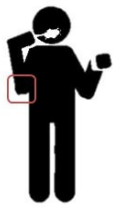

(b)
Figure 9: Toothbrushing with Different Elbow Positions:(a)High Elbow Position, (b)Low Elbow Position

\subsubsection{Statistical Features}

We also extract the statistical features from the sensor data. Given the sensor data $s=\left[s_{1}, s_{2}, \ldots s_{n}\right]$ collected in a time window, we compute their average value $\bar{s}$, variance $\sigma^{s}$ and skewness $\gamma^{s}$. In other words, we calculate the first, second and third order moments of the sensor data as the statistical features. The average value reflects the overall state of the gesture. For example, the average value of gravity data is closely related to the user's elbow position. The variance value is related to the intensity of the motions. For example, the variance of the acceleration shows how intensely the user is brushing the teeth.

The skewness is used to distinguish the subtle differences of the motions for brushing the upper or lower teeth. Skewness has been used to measure how asymmetric a random variable is about its mean. For example, when a user brushes the front upper outer surface, he generally exerts larger force when moving the toothbrush from the gum line downward to the end of the upper teeth. After that, with a smaller force, he raises the brush upward to prepare for next brushing stroke. When brushing the front lower outer surface, the process is the opposite: the toothbrush moves upward with a larger force and downward with a smaller force. The difference in the acceleration of the downward-upward motions can be captured by the skewness of the sensor data.

\subsection{Dynamic Gestures Variations}

We now consider the variation of the toothbrushing motions for the toothbrushing gestures for the same surface. We focus on the change of elbow positions during the brushing. This is illustrated in Figure 9. In this figure, the user (a) is brushing with his elbow raised up, whereas, the user (b) is brushing with his elbow put down. This type of elbow position variation is common in real life. When the user changes his or her elbow positions, the sensing results from the smartwatch change significantly, even though the user is still brushing the same tooth surface with the same technique. This type of variation can significantly affect the toothbrushing surface recognition accuracy.

This type of variation is due to the change of watch coordinates $X_{w}-Y_{w}-Z_{w}$. When the elbow position is high, the $Z_{w}$ is facing vertically upward. However, when the elbow position is low, $Z_{w}$ rotates to the horizontal right-hand side. On the other hand, since the user is brushing the same surface, the motions of the toothbrush remain the same. As a result, the sensor data collected are significantly different due to the rotation of the coordinate system.

The basic idea is to conduct a coordinate rotation transformation to offset this type of variation. We compute the rotation matrix $R$ to transform the sensor data from the smartwatch's coordinates $X_{w}-Y_{w}-Z_{w}$ to the user coordinates $X_{u}-Y_{u}-Z_{u}$. 


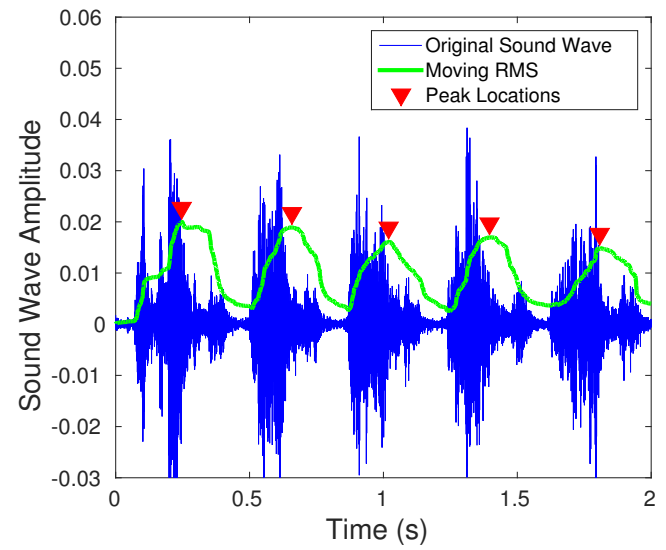

Figure 10: Brushing Stroke Estimation

To compute the rotation matrix $R$, we have the following two observations. Firstly, gravity sensed by the watch is parallel to the $Z_{u}$. This is because, by definition, $Z_{u}$ is perpendicular to the floor, and parallel to gravity. Secondly, when the user is brushing, the $Y_{w}$ direction of the watch is approximately parallel to the $Y_{u}$ direction of the user, since when the user is brushing teeth, his/her hand is held up in the front. Based on these two observations, we can compute the rotation matrix $R$ by solving the following equation set:

$$
\begin{aligned}
& {[0,0, g]=R * \text { gravity }} \\
& {[0,1,0]=I * R * \overrightarrow{Y_{w}}}
\end{aligned}
$$

Here gravity represents the gravity sensed by the watch, which can be obtained by the Android API. $g$ represents the magnitude of gravity. We take the value of $9.8 \mathrm{~m} / \mathrm{s}^{2}$. By multiplying the raw sensor data with the rotation matrix $R$, we can get the sensor data represented in the user's coordinate. After this transformation, the sensor data are ready to be used for the feature extraction algorithms.

\section{COUNTING TOOTHBRUSHING STROKES USING ACOUSTIC SENSING}

Each time a toothbrushing gesture is identified, our system estimates the number of brushing strokes conducted during that time interval. In this study, we use the acoustic sensors to achieve this goal. The acoustic sensors have been used in toothbrushing surface recognition [20]. One advantage of the acoustic sensors is that they are affected by noises different from those affecting the motion sensors. Therefore, by including the acoustic sensing in the toothbrushing monitoring, we can potentially improve the system robustness to noises.

Sounds are generated during each toothbrushing stroke. To count the toothbrushing strokes, our system use a Root Mean Square (RMS) based approach to track the intensity of the sound wave, and mark each significant peak as an indicator of a toothbrushing stroke. Given any sound wave series $q_{t}$ collected for the current time interval $\left[t_{\text {start }}, t_{\text {end }}\right]$, we compute the moving RMS value during this time window, denoted by $q_{t}^{r m s}$. After the RMS value of the sound wave is computed, we then find all the local maximas of $q_{t}^{r m s}$ that have values larger than the average RMS value of the entire window. Then we combine peaks that are within 0.1 seconds of each other. We count all the remaining sound

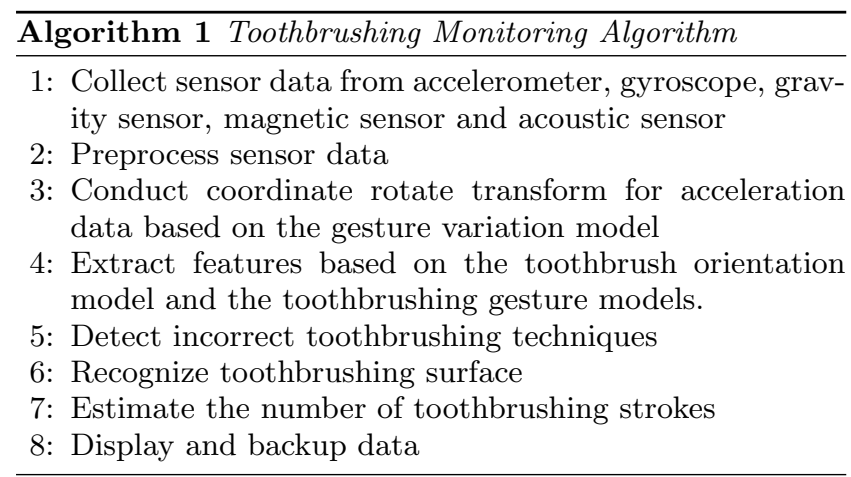

wave peaks as an estimation of toothbrushing strokes. This algorithm is illustrated in Figure 10. We can see that in this figure, the sound wave is collected in 2 seconds. There are 5 peaks of RMS value detected, which correspond to the 5 toothbrushing strokes during this time interval.

\section{TOOTHBRUSHING MONITORING ALGORITHM}

Our gesture recognition strategy is summarized in Algorithm 1. During the toothbrushing process, the smart watch continuously collects sensor data from the accelerometer, gyroscope, gravity sensor, magnetic sensor and acoustic sensor. These data are buffered in windows of a fixed time length. Then the acceleration data are processed using gesture variation offset algorithm described in Section 4.3. Based on the toothbrush orientation model and the arm motion models, the system extracts the toothbrush orientation features, the forearm rotation features, the elbow/shoulder flexion/extension features, wrist flexion/extension features, PCA-based motion direction features and the statistical features.

After all the features are extracted, the system proceeds to detect incorrect toothbrushing techniques. The incorrect toothbrushing technique detection algorithm serves two goals. Firstly, it filters out unseen gestures, so that the surface classification algorithm can have better performance. Secondly, it warns users about incorrect toothbrushing techniques from time to time.

The detection of incorrect toothbrushing technique is challenging because there are many different incorrect ways of brushing. Therefore, it is impractical to collect training data for all these techniques. Our strategy is to regard this problem as an anomaly detection problem. Based on our models of toothbrush orientations and toothbrushing gestures, our system will identify whether or not the sensor data is normal (Bass toothbrushing technique), or is too different from the training data (non-Bass toothbrushing technique).

We select the one-class Support Vector Machine (SVM) classifier to detect incorrect toothbrushing techniques. The nice property of SVM is that by using the kernel trick, the algorithm can compute a highly non-linear separating plane for classification. The one-class SVM separates all the data points from the origin in the feature space and maximizes the distance from this hyperplane to the origin [32]. This results in a binary function that returns +1 if the data is in the region of the training data points, and -1 elsewhere. Each time an incorrect toothbrushing technique is detected, a message will be displayed to notify the user. 


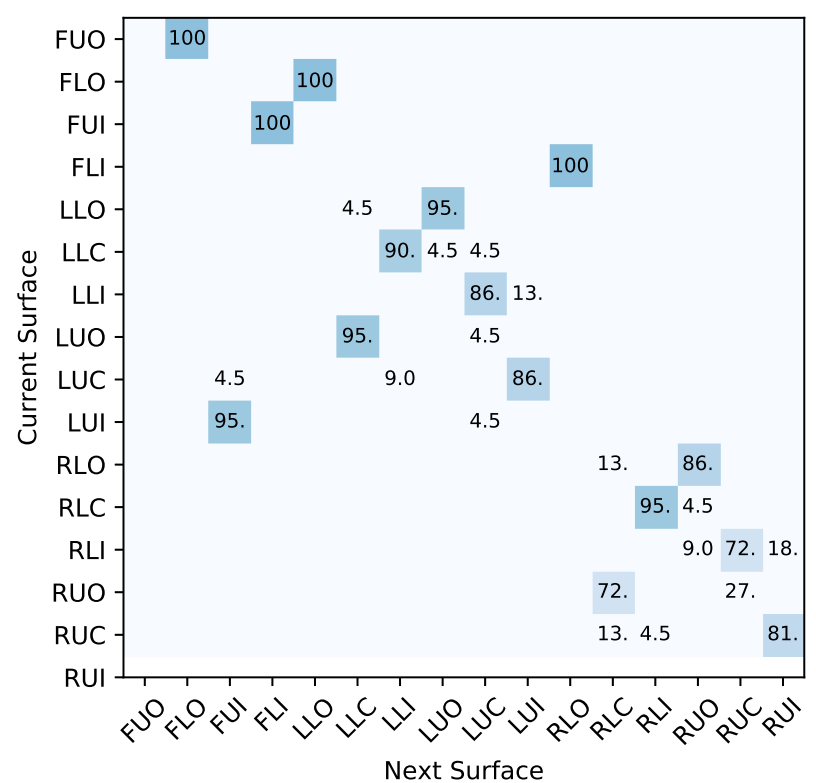

Figure 11: Transition Probability for Toothbrushing Surface

If the input data is recognized to be the correct (Bass) toothbrushing technique, the system will determine the toothbrushing surface at the next stage. In general, any multiclass classifier can be used. We evaluated several classifiers and selected the Naive Bayes classifier. Specifically, given the training label (the ground truth toothbrushing surface) $z_{i}$ and the training feature set $f_{i}$, the conditional probability $p\left(f_{i} \mid z_{i}\right)$, the class prior probability $p\left(z_{i}\right)$ and the feature probability distribution $p\left(f_{i}\right)$ are computed. In the testing phase, given an input feature set $f_{\text {inquiry }}$, the predicted class probability is computed using the Bayes Rule:

$$
p\left(z \mid f_{\text {inquiry }}\right)=\frac{p\left(f_{\text {inquiry }} \mid z_{i}\right) * p\left(z_{i}\right)}{p\left(f_{\text {inquiry }}\right)} .
$$

The label $z^{\prime}$ that maximizes $p\left(z^{\prime} \mid f_{\text {inquiry }}\right)$ is returned by the algorithm as the predicted toothbrushing gesture.

Finally, the toothbrushing stroke estimation algorithm is used. The estimated number of toothbrushing strokes and the predicted labels are recorded and will be presented to the user after the toothbrushing is finished.

\subsection{Improving Recognition Accuracy with Toothbrushing Order}

People usually have set toothbrushing habits. One such habit is the order of brushing the 16 tooth surfaces. With the data we collected, we observed that users had certain patterns in the order of toothbrushing. Similar observations are also reported in literature [25]. In this subsection, we develop a hidden Markov model to learn the probability distribution of the user-specific brushing order, which allows us to estimate the current brushing surface based on previously brushed surfaces. We can also use it to correct false recognition results from motion models. Combined with our gesture recognition algorithms, this improves the accuracy of our brushing gestures recognition solution.

\subsubsection{Observations}

Some users have stable toothbrushing orders. As an example, we analyzed twenty-two toothbrushing sessions for one of the users, and computed the transition probability matrix of the brushing order in Figure 11. We found that there was a strong pattern in the toothbrushing order. From this matrix, we can see that the transition probabilities from Front Upper Outer (FUO) to Front Lower Outer (FLO), from FLO to Left Lower Outer (LLO), from Front Upper Inner (FUI) to Front Lower Inner (FLI), and from FLI to Right Lower Outer (RLO), are $100 \%$. This means that these orders were followed in all the twenty-two toothbrushing sessions. Besides, for all the surfaces, the transition probabilities for the most likely next surface are all greater than $70 \%$. In sum, the toothbrushing order is highly predictable and was followed by most of the twenty-two toothbrushing sessions.

\subsubsection{Integration with the Surface Recognition Algorithm}

We design a simple algorithm that can be easily integrated with our system. The algorithm maintains a surface transition probability matrix $M_{t}$. The algorithm is invoked only when the Bayes based surface classifier makes a prediction $z(t)^{\prime}$ that is different from the previous prediction $z(t-1)$. If the classification confidence $p\left(z(t)^{\prime} \mid f\right)$ is greater than an ambiguity threshold $T_{c}$, it means the predication is accurate, and the new prediction value $z(t)^{\prime}$ will be used to update the matrix $M_{t}$. On the other hand, if the classification confidence $p\left(z(t)^{\prime} \mid f\right)$ is smaller than $T_{c}$, the algorithm will find out the most likely next surface $z^{\prime \prime}$ given the previous surface $z(t-1)$ from the transition matrix $M_{t}$. Then the algorithm computes the weighted probability $c_{1}=(1-\alpha) p\left(z(t)^{\prime} \mid f\right)+\alpha M_{t}\left(z(t-1), z(t)^{\prime}\right)$ and $c_{2}=(1-\alpha) p\left(z^{\prime \prime} \mid f\right)+\alpha M_{t}\left(z(t-1), z^{\prime \prime}\right)$. The algorithm will return $z(t)^{\prime}$ if $c_{1} \geq c_{2}$, and return $z^{\prime \prime}$ otherwise.

\section{EVALUATION}

\subsection{Experiment Setup}

\subsubsection{System Implementation}

We have implemented and tested the system on the Gear Live smartwatch [4], Nexus 7 (2013) tablets [3] and the Amazon Web Service (AWS) [1] platform. The Gear Live smartwatch has an accelerometer, a gyroscope, a gravity sensor, a magnetic sensor, and a microphone. It has $512 \mathrm{Mb}$ RAM and a Quad-core 1.3GHz CPU. It uses a Bluetooth radio to communicate with the tablet. We use the AWS S3 service to store the sensor data, and use the AWS EC2 computation platform to process the data.

For the software part, we have implemented the feature extraction and classification modules on the Android Operating System installed on the Gear Live smartwatch.

\subsubsection{Datasets}

We deployed this system to 12 volunteers to conduct daily toothbrushing. At the beginning of the experiment, we demonstrated the Bass technique to the volunteers in person and let them watch an instruction video. Each volunteer brushed his or her teeth for at least two minutes twice a day. The system asked users to label each of their brushing surfaces with a user interface on the tablet during each toothbrushing session. This baseline collection phase lasted for up to 14 days. After this phase, the users conducted toothbrushing normally wearing our wrist watch without having to input gesture labels. The ground truth labels were 
recorded by a second observer or by video recording. The second phase of data collection lasted for up to 7 days. By one set of training data, we mean the amount of sensor data collected in one toothbrushing session.

To evaluate the incorrect toothbrushing detection algorithm, we recorded sensor data from four of these volunteers. The volunteers brushed their teeth using the horizontal technique, one of the most commonly seen incorrect toothbrushing technique [28]. They brushed each of their inner and outer surfaces (12 surfaces) using horizontal strokes. Each volunteer brushed for five times. The incorrect toothbrushing detection algorithm was trained solely by correct toothbrushing data and was tested using both the correct and incorrect toothbrushing data.

\subsubsection{Evaluation Methodology}

Toothbrushing sensor data collected from each user are divided into one-second segments. We use k-fold cross validation for performance evaluation. Each round of crossvalidation involves partitioning a sample of data into complementary subsets, performing the analysis on one subset (called the training set), and validating the analysis on the other subset (called the validation set or testing set). To obtain statistical confidence, ten rounds of cross-validation were performed using different partitions, and the validation results are averaged over the rounds.

To measure the toothbrushing surface recognition accuracy, we use the precision, recall, and $F_{1}$ score. Precision is defined as the percentage of time the algorithm makes correct predictions. The recall rate is defined as the percentage of time when the user is brushing any surface, and the system recognizes it correctly. $F_{1}$ score is commonly used to integrate both results. Specifically, precision, recall and $F_{1}$ score are computed using the following equation:

$$
\begin{aligned}
& \text { precision }=T P /(T P+F P) \\
& \text { recall }=T P /(T P+F N) \\
& F_{1}=2 * \text { precision } * \text { recall } /(\text { precesion }+ \text { recall })
\end{aligned}
$$

In these equations, TP represents true positive, FP represents false positive and $\mathrm{FN}$ represents false negative.

To evaluate the incorrect toothbrushing gesture recognition accuracy, we use the Recall rate and the True Negative Rate (TNR). TNR measures the proportion of incorrect toothbrushing gestures that are detected by the algorithm. It is defined as $T N /(T N+F P)$.

\subsection{Toothbrushing Surface Recognition}

\subsubsection{The Effectiveness of Different Features}

We present the results on effectiveness of different features. Specifically, we evaluated the statistical features (sta) defined in Section 4.2.2, the gesture model features (model) defined in Section 4.1, the PCA direction features (pca) defined in Section 4.2.1, and the toothbrush bristle orientation features (mag) defined in Section 3.4. Finally, we also tested the effectiveness of the toothbrushing order based algorithm described in Section 6.1. In each round of cross validation, three sets of sensor data were used to train the machine learning algorithm, and the remaining sensor data were used for evaluation. The evaluation accuracy results are shown in Figure 12.

From this figure, we can see that when only the statistical

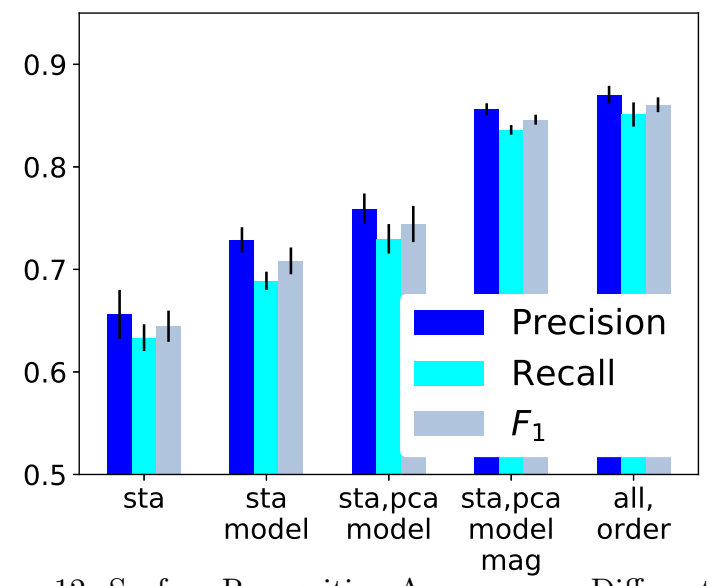

Figure 12: Surface Recognition Accuracy vs. Different Feature Sets

features are used, the recognition precision is only $65.6 \%$, meaning that the subtle differences of the motions are not captured by these features alone. When the gesture model features are included, the system can better distinguish the back-forth horizontal motions and the vertical rolling motions. As a result, the recognition precision is improved to $72.9 \%$. The PCA features are designed to determine the directions of the toothbrush motions, and can provide an additional $3 \%$ of improvements to the recognition precision.

When the magnetic sensing based toothbrush orientation features are used, the precision is boosted to $85.6 \%$. The improvement is due to the features' effectiveness in distinguishing orientations of the toothbrushes, which are hard to recognize using motion sensors alone. For example, without these features, the toothbrushing gestures of upper or lower chewing surfaces, which have almost identical motions, are hard to distinguish.

In the implementation of the toothbrushing order based algorithm, we set the ambiguity threshold $T_{c}$ to a high value so that only approximately $5 \%$ of the testing data will be recognized as ambiguous. The results are shown in Figure 12 in the column with label "all,order". From this figure, we can see that there is a $2.1 \%$ increase in the surface recognition precision. It shows that toothbrushing order information is useful in the surface recognition algorithm. Nevertheless, from this experiment we observed that there were still two ways to improve the algorithm. Firstly, some false predictions occur continuously. In such cases, the algorithm would falsely regard them as brushing the same surface so that no correction will occur. Secondly, we find that the posterior probability of the Naive Bayes classifier is not always an effective indicator of prediction confidence. In some cases, the prediction confidence (i.e. the posterior probability) returned by the Bayes classifier is close to 1 , even when the prediction results are incorrect [8]. To fully utilize the toothbrushing order information, it's important to have a more accurate prediction confidence estimation.

We note that in different rounds of cross validation, about $3 \%$ of standard deviation in the recognition accuracy (precision, recall and $F_{1}$ ) existed, as we can see in the figure. To improve the confidence of the recognition accuracy results, we have conducted ten rounds of experiments and calculated the average.

\subsubsection{Surface Recognition Confusion Matrix}




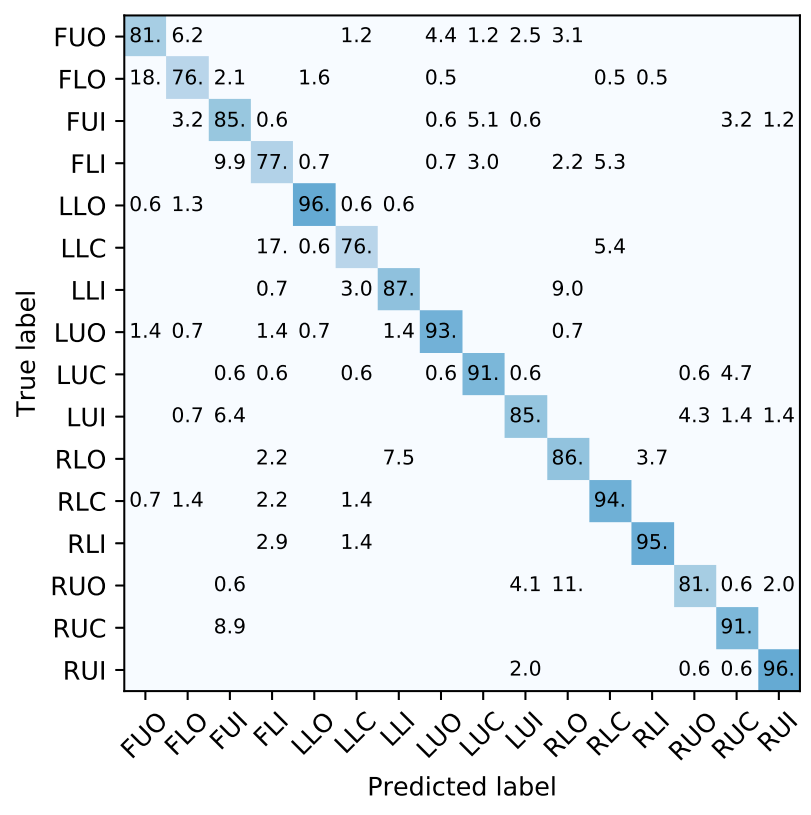

Figure 13: Toothbrushing Surface Recognition Confusion Matrix

To better understand the strengths and weaknesses of the surface recognition algorithm, we study the confusion matrix $M$ shown in Figure 13. In this matrix, each entry in cell $M(i, j)$ represents the probability for the algorithm to predict a gesture as label $j$, when its true label is $i$. Due to the roundup error, each row of the matrix may not sum up to $100 \%$.

The confusion matrix shows that our designs are effective in many ways. Firstly, we can see that our system effectively distinguishes the upper and lower chewing surfaces. For example, there are almost no confusions between RLC and RUC, or between LLC and LUC. This is because of the introduction of the magnetic sensing design, which reliably distinguish upper or lower chewing surfaces. Besides, the Left Lower Outer (LLO) surface is reliably recognized (96\%), showing the effectiveness of the forearm rotation model. Furthermore, we can see that the six elbow motion gestures (FUI, FLI, LLC, LUC, RLC, RUC) are almost never confused with other gestures. This also shows the effectiveness of the elbow motion model.

We can also see that there are difficulties in toothbrushing surface recognition. There are a few toothbrushing gestures that are very similar to each other. We can see that there are $18 \%$ of Front Lower Outer (FLO) data falsely classified as Front Upper Outer (FUO). Gestures of toothbrushing these two surfaces are very similar, and the major difference is the rolling direction of the toothbrush, and the skewness features(Section 4.2.2) are designed to distinguish them. Nevertheless, there are still cases not recognized. Another major source of error comes from the toothbrushing direction recognition. To name a few, $17 \%$ of Left Lower Chewing (LLC) data are falsely classified as Front Lower Inner (FLI), and $8.1 \%$ of Right Upper Chewing (RUC) data are classified as Front Upper Inner (FUI). The motions of these three gestures are similar. They are all horizontal back-forth motions primarily driven by elbow/shoulder flexion/extension. The key to distinguishing them is to recognize their motion

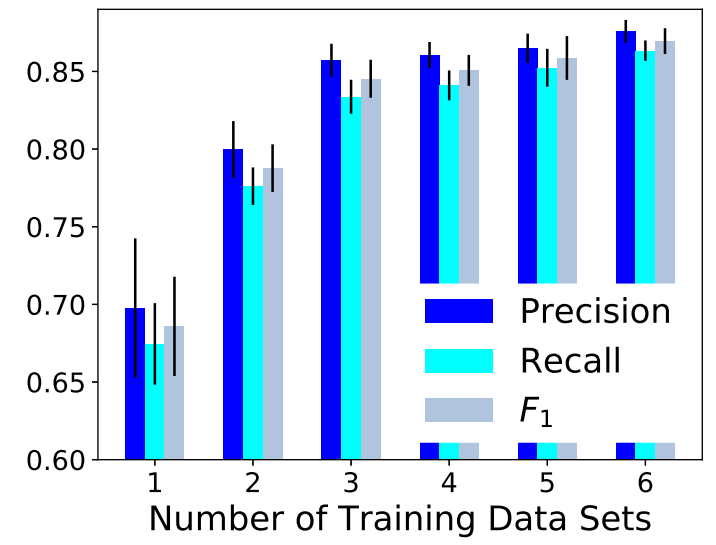

Figure 14: Surface Recognition Accuracy vs. Training Data Set Size

directions, and the PCA-based direction features are used. However, there are still cases when this feature cannot distinguish effectively.

Even though there are recognition errors during some onesecond segments, since the brushing of each surface lasts for several seconds, it is still possible for the system to recognize the surface accurately. We will work on this direction in the future work.

\subsubsection{The Influence of Training Data Set Size}

In this experiment, we studied the toothbrushing surface recognition accuracy when different amount of training data were used. For each user, we use from one to six sets of toothbrushing data to train the machine learning model. After the model had been trained, we evaluated its recognition accuracy using all the remaining sensor data. The results are shown in Figure 14.

From this figure, we can see that when one set of toothbrushing data is used, the surface recognition precision is $69.2 \%$. As more training data sets are used, the surface recognition accuracy improves. When the number of training data sets is three, the recognition precision, recall and $F_{1}$ score are $85.6 \%, 83.6 \%$, and $84.5 \%$, respectively. When more than three sets of sensor data are used, the recognition accuracy remains stable, meaning that the marginal gain in the system performance is small. This experiment shows that to have high-quality toothbrushing monitoring, a user just needs to brush his or her teeth carefully for three times to allow the system to learn. After that, the system will be able to provide high-quality monitoring.

\subsection{Incorrect Toothbrushing Technique Detection}

The evaluation results are shown in Figure 15. We can see that when only one set of toothbrushing data is used in training, the recall rate is $74 \%$, and the TNR is $50 \%$. As the number of training data sets increases to 4 , the recall rate stabilizes at $90 \%$, while the TNR gradually increases to $66 \%$. When at least four sets of training data are provided, the system can maintain a high recall rate of $90 \%$. Since the recall rate is high, the users will be able to brush continuously without being disturbed by false alarms frequently, as long as they are brushing using the Bass technique.

We can also see that the true negative rate ranges from 


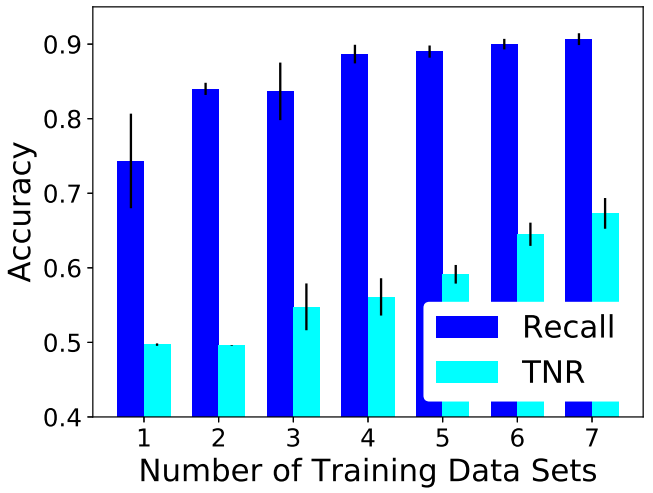

Figure 15: Incorrect Toothbrushing Technique Detection

\begin{tabular}{|c|c|c|}
\hline & Avg. (mAh) & Std. Dev. (mAh) \\
\hline \hline Training & 3.3 & 0.63 \\
\hline Monitoring & 3.9 & 0.60 \\
\hline Google Fit & 2.8 & 0.53 \\
\hline
\end{tabular}

Table 1: Energy Consumption for Each Toothbrushing Session

$50 \%$ to $66 \%$, meaning that the algorithm can miss incorrect brushing techniques. Although the system may miss incorrect gestures from time to time, its detection accuracy is high enough to detect long-term incorrect toothbrushing habits. This is because each time the user brushes in the incorrect technique for one second, the system on average has a probability of $66 \%$ of detecting it. If the incorrect toothbrushing technique is frequently repeated, the probability of the system identifying at least one instance of such incorrect brushing goes up.

\subsection{Impact on Battery Life}

One resource constraint of our system is in the smartwatch because it has smaller battery capacity $(310 \mathrm{mAh})$. In this section, we conducted experiments to test the impact of our system on the watch battery life. Using three sets of the toothbrushing monitoring system, we repeated the toothbrushing monitoring process for five times each, and measured the energy consumptions using the battery historian tool [2]. The results are shown in Table 1. During the training phase, the system did not execute recognition algorithms. Instead, the system recorded sensor data and uploaded the data to the cloud after each toothbrushing session. In the monitoring phase, besides recording and uploading sensor data, the system also conducted recognition algorithms in real-time every second, and sent the recognition results to the tablet for display. We can see that under the highest workload, the smartwatch consumed 3.9 mAh per toothbrushing session. Since each user brushed for about 2 minutes for twice a day, normal daily toothbrushing with our system consumes less than $5 \%$ of the smartwatch battery capacity.

We also tested the energy consumption rate of another commonly used activity recognition app for smartwatch, the Google Fit. This app continuously recorded the value of the step sensor and display the results. We run this app on three smartwatches for five times, and each time lasted for two minutes. We found that the smartwatches on av-

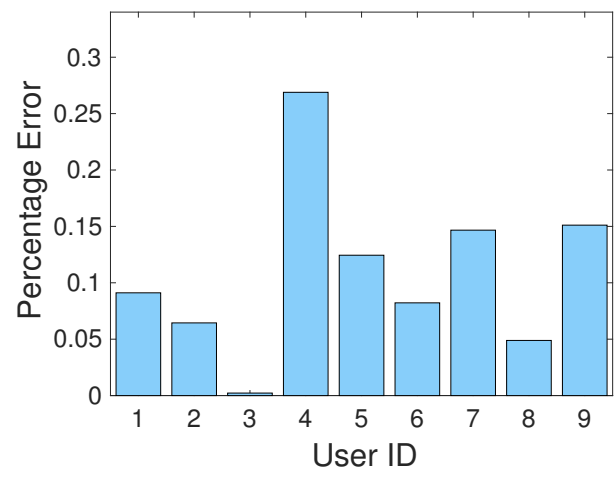

Figure 16: Stroke Counting Accuracy

erage consumed $2.8 \mathrm{mAh}$, with standard deviation of 0.53 $\mathrm{mAh}$. A possible reason for the lower energy consumption was that fewer computation tasks were executed than our system. Besides, we also tested the idle energy consumption of the smartwatch. When a smartwatch was left unattended to display time only, the battery life was about 110 hours. These results showed that in terms of energy consumption, our system had a similar performance to those in the market, and would not seriously affect the smartwatch battery life.

\subsection{Case Study}

To evaluate the effectiveness of our smart toothbrushing system in improving dental hygiene, we have recruited 12 volunteers and conducted a user study. We invited a dentist from a local hospital to check plaque level of every tooth surface for each volunteer. Each subject chewed a plaque disclosing pill, which highlighted plaques on teeth. The dentist recorded the plaque levels using the Turesky Modified Quigley-Hein Plaque Indexes (TQHPI) [12] before and after the subjects brushed their teeth. Each subject was given the instructions on the Bass technique and was using our system during toothbrushing.

This study consisted of two parts. In the first experiment, 9 volunteers brushed each of their tooth surfaces with thirty brushing strokes. We evaluated the effectiveness of our system in estimating toothbrushing effort. In the second experiment, 3 volunteers brushed teeth with different numbers of strokes for each surface. We assessed the relation between toothbrushing effort and the plaque removal quality.

We note that the result of this set of experiments can be affected by a few factors, such as insufficient chewing of plaque disclosing tablets, personal toothbrushing habit, and amount of toothpaste. Fortunately, the obtained result was consistent and therefore served the purpose of demonstrating the potential value of our smart toothbrushing system.

\subsubsection{Counting Toothbrushing Stokes}

In the first experiment, we tested the effectiveness of the toothbrushing stroke counting algorithm described in Section 5. The detection error was defined as |predicted number - ground truth|/|ground truth|.

The results are shown in Figure 16. For the 9 subjects, our algorithm on average achieves an error rate of $10.3 \%$. This demonstrates its effectiveness in evaluating the toothbrushing effort of the users.

For the subject 4, we can see that the error rate is as high as $30 \%$. This is because the subject 4 was brushing teeth 


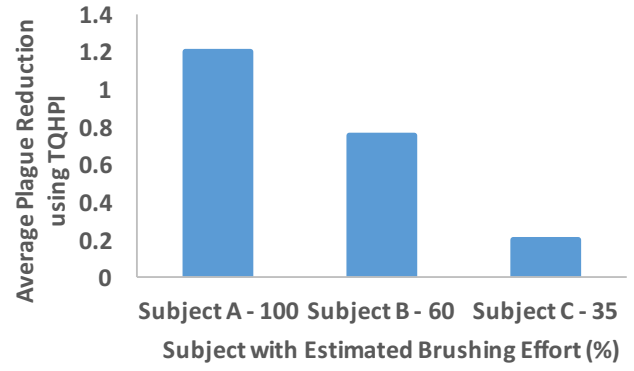

Figure 17: Tooth Plague Reduction vs. Measured Tooth Brushing Effort

with his mouth closed. As a result, the sound collected by the device was small and had low signal to noise ratio. To solve this problem, we plan to design a hybrid toothbrushing stroke counting algorithm that combines both acoustic sensing and motion sensing, which has better noise robustness.

\subsubsection{Plaque Reduction}

From the previous experiment, we showed that our system was able to count the toothbrushing strokes with small error $(10.3 \%)$. In the second experiment, we tested how the number of toothbrushing strokes were related to the tooth plaque removal rate. 3 subjects were asked to perform toothbrushing with different numbers of brushing strokes (10, 20, and 30 ) on each surface, while they used our system to estimate their brushing effort. Our system estimated the effort of toothbrushing by counting the number of brushing strokes. 30 strokes on each surface was considered $100 \%$ brushing effort in this analysis, the tests of 10 and 20 brushes on each surface were normalized accordingly.

The experimental result of this test is shown in Figure 17. We can see that the plague reduction increases as the effort of toothbrushing increases. Insufficient toothbrushing subject B ( $60 \%$ effort) and subject $\mathrm{C}$ (35\% effort) only remove $62.7 \%$ and $16.8 \%$ plague, comparing to that of subject A (100\%). These results show that first insufficient toothbrushing can be reliably detected by our system. And second, insufficient toothbrushing directly affect the effectiveness of the plaque removal. Even short term insufficient toothbrushing activities do harm to our teeth as plaque accumulates quickly and introduces gum diseases.

\section{STATE OF THE ART}

Various techniques have been employed to perform gesture recognition, such as radio waves [30], video [24], and wearable sensors [9, 19, 31]. Among different wearable sensing devices, wrist watch has received a lot of attention. In paper $[14,22]$, the authors studied the hand hygiene quality assessment using wrist-worn devices. They achieved good performance in recognizing correct hand-washing gestures. The authors of [27] designed probabilistic models to successfully recognize smoking gestures with high accuracy. In [23], the authors applied Dynamic Time Warping (DTW) to match the inquiry data to the model data in the training sets. They achieved good performance (over $98 \%$ of accuracy) in classifying eight gestures. These works provided valuable insights and demonstrated the feasibility of complex gesture recognition using wrist-worn devices. However, their primary goal was to conduct gesture recognition. In our work, besides recognition of gestures, we also assessed the quality of toothbrushing. Moreover, we developed a novel magnetic sensing algorithm to capture the motion of a hand tool, the toothbrush.

In order to monitor toothbrushing, several systems have been designed. In [10], authors designed a vision-based motion tracker for toothbrushing. In [20], the authors developed a hidden Markov model to recognize acoustic patterns during toothbrushing. Different from these works, we mainly focused on motion recognition for both toothbrush and brushing gestures. Also, our approach recognized all 16 tooth surfaces with high accuracy, whereas their solution only recognized four different tooth quads.

There have been many research works on electric toothbrushes. In [33], the authors presented evidence that Oscillating Rotating Pulsating toothbrushes achieved effective hygiene result. The authors of [34] explored the use of pressure sensors on the electric toothbrushes. The authors of [21] used acceleration and magnetic sensors in the electric toothbrush to detect the motion and orientation of the toothbrush. Our magnetic sensing design was related to but different from the designs proposed in these works. To sense the motions of the toothbrush, previous approaches generally installed various sensors on the toothbrush, while our approach was to install a magnet on the toothbrush to make it sensible by the magnetic sensor on the smartwatch. Our approach significantly reduced the cost and the system complexity, and improved the system robustness to the change of geomagnetic field. Besides the novelty in design, our system also tracked the toothbrushing quality and detected incorrect toothbrushing gestures, which were not addressed in previous studies.

\section{CONCLUSION AND FUTURE WORK}

As the most important oral hygiene activities at home, daily toothbrushing can effectively prevent tooth decay and gum diseases. To accurately monitor toothbrushing compliance and effectiveness, we developed a novel sensing system using an off-the-shelf toothbrush and a wrist watch. Experimental results with 12 users for up to 3 weeks showed that our solution reliably recognized the gestures for all 16 brushing surfaces using the Bass technique with a high accuracy.

In the future, we plan to investigate the recognition of different incorrect toothbrushing, which is important to help users form good toothbrushing habits. We will apply the deep learning based algorithms to capture the subtle changes in the data from the magnetic sensor and gravity sensor due to the incorrect toothbrushing gestures. We are working with the dental professionals to conduct a rigorous clinical study on the effectiveness of our system with real patients. We will conduct a control group study, where one group of users will brush teeth with the help of our system, while the others will brush normally. By comparing the plaque removal quality in both groups, we can show whether or not the system can improve dental hygiene.

\section{ACKNOWLEDGMENTS}

This work is supported in part by NSF grants CNS 1536086, CNS 1463722, IIS 1460370 and CNS 1553273. We thank the anonymous reviewers and our shepherd, Lama Nachman, for their insightful comments. We are grateful to Guoliang Xing, John Stankovic, Jie Gao, Wenting Lin D.M.D. and Maria Ryan D.M.D. for valuable discussions. 


\section{REFERENCES}

[1] Amazon web service. https://aws.amazon.com. Accessed: 2016-08-24.

[2] The battery historian. https://github.com/google/battery-historian. Accessed: 2016-09-11.

[3] Nexus 7(2013). https://www.asus.com/us/Tablets/Nexus_7_2013/. Accessed: 2016-08-24.

[4] Samsung gear live. http://www.samsung.com/us/ support/gear/gear-live-support/. Accessed: 2016-08-24.

[5] H. Abdi and L. J. Williams. Principal component analysis. Wiley Interdisciplinary Reviews: Computational Statistics, 2(4):433-459, 2010.

[6] A. D. Association. Tackling tooth decay. Journal of American Dental Association, 2013.

[7] D. Bartlett and P. Shah. A critical review of non-carious cervical (wear) lesions and the role of abfraction, erosion, and abrasion. Journal of Dental Research, 85(4):306-312, 2006.

[8] P. N. Bennett. Assessing the calibration of naive bayes posterior estimates. Technical report, DTIC Document, 2000.

[9] S. Berman and H. Stern. Sensors for gesture recognition systems. Systems, Man, and Cybernetics, Part C: Applications and Reviews, IEEE Transactions on, 2012.

[10] Y.-C. Chang, J.-L. Lo, C.-J. Huang, N.-Y. Hsu, H.-H. Chu, H.-Y. Wang, P.-Y. Chi, and Y.-L. Hsieh. Playful toothbrush: Ubicomp technology for teaching tooth brushing to kindergarten children. In Proceedings of the SIGCHI Conference on Human Factors in Computing Systems, CHI '08, 2008.

[11] H.-T. Cheng, F.-T. Sun, M. Griss, P. Davis, J. Li, and D. You. Nuactiv: Recognizing unseen new activities using semantic attribute-based learning. In Proceeding of the 11th Annual International Conference on Mobile Systems, Applications, and Services, MobiSys '13, pages 361-374, New York, NY, USA, 2013. ACM.

[12] M. Cugini, M. Thompson, and P. Warren. Correlations between two plaque indices in assessment of toothbrush effectiveness. J Contemp Dent Pract, $7(5): 1-9,2006$.

[13] C. for Disease Control and Prevention. National center for health statistics. Health, United States, 2014.

[14] V. Galluzzi, T. Herman, and P. Polgreen. Hand hygiene duration and technique recognition using wrist-worn sensors. In Proceedings of the 14 th International Conference on Information Processing in Sensor Networks, IPSN '15, pages 106-117, New York, NY, USA, 2015. ACM.

[15] C. Ganss, N. Schlueter, S. Preiss, and J. Klimek. Tooth brushing habits in uninstructed adults: frequency, technique, duration and force. Clinical oral investigations, 13(2):203-208, 2009.

[16] J. Gibson and A. B. Wade. Plaque removal by the bass and roll brushing techniques. Journal of periodontology, 48(8):456-459, 1977.

[17] M. Hammadeh and J. Rees. The erosive susceptibility of cervical versus occlusal enamel. The European journal of prosthodontics and restorative dentistry,
9(1):13-17, 2001.

[18] I. Jolliffe. Principal Component Analysis. John Wiley \& Sons, Ltd, 2014.

[19] B. Kellogg, V. Talla, and S. Gollakota. Bringing gesture recognition to all devices. In 11th USENIX Symposium on Networked Systems Design and Implementation (NSDI 14), 2014.

[20] J. Korpela, R. Miyaji, T. Maekawa, K. Nozaki, and H. Tamagawa. Evaluating tooth brushing performance with smartphone sound data. In Proceedings of the 2015 ACM International Joint Conference on Pervasive and Ubiquitous Computing, pages 109-120. ACM, 2015.

[21] Y. J. Lee, P. J. Lee, K. S. Kim, W. Park, K. D. Kim, D. Hwang, and J. W. Lee. Toothbrushing region detection using three-axis accelerometer and magnetic sensor. IEEE Transactions on Biomedical Engineering, 2012.

[22] Y. Li, X. Chen, X. Zhang, K. Wang, and Z. Wang. A sign-component-based framework for chinese sign language recognition using accelerometer and semg data. Biomedical Engineering, IEEE Transactions on, 59(10):2695-2704, Oct 2012.

[23] J. Liu, L. Zhong, J. Wickramasuriya, and V. Vasudevan. uwave: Accelerometer-based personalized gesture recognition and its applications. Pervasive and Mobile Computing, 5(6):657 - 675, 2009. PerCom 2009.

[24] R. Lockton and A. W. Fitzgibbon. Real-time gesture recognition using deterministic boosting. In in Proceedings of the British Machine Vision Conference (BMVC 'O2), 2002.

[25] I. MacGregor and A. Rugg-Gunn. A survey of toothbrushing sequence in children and young adults. Journal of periodontal research, 14(3):225-230, 1979.

[26] R. S. Manly and F. Schickner. Factors influencing tests on the abrasion of dentin by brushing with dentifrices. Journal of Dental Research, 23(1):59-72, 1944.

[27] A. Parate, M.-C. Chiu, C. Chadowitz, D. Ganesan, and E. Kalogerakis. Risq: Recognizing smoking gestures with inertial sensors on a wristband. In Proceedings of the 12th Annual International Conference on Mobile Systems, Applications, and Services, MobiSys '14, pages 149-161, New York, NY, USA, 2014. ACM.

[28] B. T. Piotrowski, W. B. GILLETTE, and E. B. HANCOCK. Examining the prevalence and characteristics of abfractionlike cervical lesions in a population of us veterans. The Journal of the American Dental Association, 132(12):1694-1701, 2001.

[29] M. Poyato-Ferrera, J. Segura-Egea, and P. Bullón-Fernández. Comparison of modified bass technique with normal toothbrushing practices for efficacy in supragingival plaque removal. International journal of dental hygiene, 1(2):110-114, 2003.

[30] Q. Pu, S. Gupta, S. Gollakota, and S. Patel. Whole-home gesture recognition using wireless signals. In Proceedings of the 19th Annual International Conference on Mobile Computing Ë\#38; Networking, MobiCom '13, 2013.

[31] R. I. Ramos-Garcia and A. W. Hoover. A study of 
temporal action sequencing during consumption of a meal. In Proceedings of the International Conference on Bioinformatics, Computational Biology and Biomedical Informatics, BCB'13, pages 68:68-68:75, New York, NY, USA, 2013. ACM.

[32] B. Schölkopf, R. C. Williamson, A. J. Smola, J. Shawe-Taylor, J. C. Platt, et al. Support vector method for novelty detection. In NIPS, volume 12 , pages 582-588, 1999.

[33] O.-B. E. Toothbrushes. The scientific evidence behind oscillating-rotating-pulsating brush technology. 2013.

[34] P. Walters, R. Bartizek, and A. Biesbrock. A study to assess the use of the pressure sensor in the oral-b triumph with smartguide. 2008. 